\title{
Characterization and anticancer potential of ferulic acid-loaded chitosan nanoparticles against ME-180 human cervical cancer cell lines
}

\author{
Richa Panwar ${ }^{1}$ - Asvene K. Sharma ${ }^{1} \cdot$ Mandeep Kaloti $^{2}$. \\ Dharm Dutt $^{3} \cdot$ Vikas Pruthi $^{1}$
}

Received: 10 March 2015/Accepted: 22 September 2015/Published online: 3 October 2015

(C) The Author(s) 2015. This article is published with open access at Springerlink.com

\begin{abstract}
Ferulic acid (FA) is a widely distributed hydroxycinnamic acid found in various cereals and fruits exhibiting potent antioxidant and anticancer activities. However, due to low solubility and permeability, its availability to biological systems is limited. Non-toxic chitosan-tripolyphosphate pentasodium (CS-TPP) nanoparticles (NPs) are used to load sparingly soluble molecules and drugs, increasing their bioavailability. In the present work, we have encapsulated FA into the CS-TPP NPs to increase its potential as a therapeutic agent. Different concentrations of FA were tested to obtain optimum sized FA-loaded CS-TPP nanoparticles (FA/CS-TPP NPs) by ionic gelation method. Nanoparticles were characterized by scanning electron microscopy, Fourier transformation infrared spectroscopy (FTIR), thermogravimetric analyses and evaluated for their anticancer activity against ME-180 human cervical cancer cell lines. The FTIR spectra confirmed the encapsulation of FA and thermal analysis depicted its degradation profile. A concentration-dependent relationship between FA encapsulation efficiency and FA/ CS-TPP NPs diameter was observed. Smooth and spherical FA-loaded cytocompatible nanoparticles with an average
\end{abstract}

Electronic supplementary material The online version of this article (doi:10.1007/s13204-015-0502-y) contains supplementary material, which is available to authorized users.

Vikas Pruthi

vikasfbs@gmail.com; vikasfbs@iitr.ernet.in

1 Department of Biotechnology, Indian Institute of Technology Roorkee (IIT R), Roorkee, Uttarakhand 247667, India

2 Centre for Nanotechnology, Indian Institute of Technology Roorkee, Roorkee, Uttarakhand, India

3 Department of Paper Technology, Indian Institute of Technology Roorkee, Roorkee, Uttarakhand, India diameter of $125 \mathrm{~nm}$ were obtained at $40 \mu \mathrm{M}$ FA conc. The cytotoxicity of $40 \mu \mathrm{M}$ FA/CS-TPP NPs against ME-180 cervical cancer cell lines was found to be higher as compared to $40 \mu \mathrm{M}$ native FA. Apoptotic morphological changes as cytoplasmic remnants and damaged wrinkled cells in ME-180 cells were visualized using scanning electron microscopic and fluorescent microscopic techniques. Data concluded that chitosan enveloped FA nanoparticles could be exploited as an excellent therapeutic drug against cancer cells proliferation.

Keywords Ferulic acid · FTIR - Nanoparticles . Cytocompatible $\cdot$ Chitosan $\cdot$ Scanning electron microscopy

\section{Introduction}

Ferulic acid (FA) (4-hydroxy-3-methoxycinnamic acid) is the most abundant hydroxycinnamic acid found in plant cell walls forming covalent ester linkages to polysaccharides and ether or ester linkages to lignin. It is reported to have antioxidant, antimicrobial, anti-inflammatory, cholesterol-lowering and anticancer activities, as well as ability to prevent thrombosis and atherosclerosis (Wilson et al. 2007; Akihisa et al. 2000; Ou et al. 1999; Kayahara et al. 1999; Mori et al. 1999). Being capable of absorbing UV radiations, FA is finding place as a photoprotective constituent in many skin lotions and sunscreens (Saija et al. 2000). The strong antioxidant character of FA is attributed to its unsaturated side chain and phenolic nucleus that spontaneously forms resonance stabilized structures, thus making it an effective antiproliferative agent. FA is a dietary phytochemical that is known to possess antitumor activities against several types of cancers such as colon, gastric, breast and cervical cancers (Janicke et al. 2011; 
Kampa et al. 2004; Kyoungho et al. 2001). Approximately 500,000 cases of cervical cancer are diagnosed per year making it the second leading cause of cancer mortality in women following breast cancer (Ellenson and Wu 2004). Radiotherapy is the major therapeutic technique to minimize the effect of cervical cancer but due to its adverse effect on normal tissues, alternative plant-derived therapeutic drugs are being tested increasingly (Seiwert et al. 2007). A large number of natural compounds have shown cytotoxic effects in different cancer models either alone or together with radiation (Garg et al. 2005). Effects of FA on human cervical carcinoma cells ME-180 and HeLa have been studied and indicated that FA treatment significantly decreased radiation surviving fraction of the cancer cells and increased the lipid peroxidation indices (Subburayan et al. 2011).

Despite having valuable health benefits, bioavailability and clinical efficacy of FA remain substantially limited owing to its lower solubility and permeability in aqueous medium as compared to other solvents such as ethanol, acetone and dimethylsulphoxide (Munin and Lévy 2011; Soobrattee et al. 2005). Attempts have been made to increase its solubility and bioavailability through encapsulation in biodegradable polymeric nanoparticles (Vemula et al. 2006; Salmaso et al. 2007). Chitosan (CS), the deacetylated form of chitin (2-amino-2-deoxy-(1-4)-Dglucopyranan), exhibits excellent biodegradability, biocompatibility and antimicrobial activity (Liu et al. 2006); thus, CS nanoparticles are extensively used to deliver hydrophobic compounds including drugs, vitamins, proteins, nutrients and phenolics into the biological systems (Hu et al. 2008; Jang and Lee 2008). CS acts as a wall/shell material to envelop these compounds bearing multiple negative charges via cationic crosslinking to generate stable, non-toxic, biodegradable nanosized particles (Keawchaoon and Yoksan 2001). CS forms inter- and intramolecular cross-linkages (called ionic gelation) when comes in contact with specific polyanions such as tripolyphosphate pentasodium (TPP) for drug encapsulation (Sofia et al. 2008). Woranuch and Yoksan (2013) studied the thermal stability of eugenol by encapsulation into CS-TPP nanoparticles. Similarly, Sofia et al. (2008) synthesized CS-TPP nanoparticles loaded with tea catechins by the ionotropic gelation method.

To our best knowledge, present investigation is the first report on in vitro antiproliferative potential of FA/CS-TPP NPs against ME-180 human cervical cancer cell lines, their encapsulation efficiency, loading capacity and particles morphology. Physical states of native as well as encapsulated FA were determined by thermal studies and the interaction between active groups of chitosan nanoparticles and FA was determined by Fourier transformation infrared spectroscopy (FTIR).

\section{Materials and methods}

\section{Materials}

Low molecular weight chitosan (85\% deacetylation degree), TPP, heat inactivated fetal calf serum (FBS), glutamine, penicillin-streptomycin, EDTA and trypsin were purchased from (Sigma Chemicals Co., St. Louis, USA). FA, cell culture-grade dimethyl sulfoxide (DMSO), acridine orange $(\mathrm{AO})$, ethidium bromide $(\mathrm{EtBr})$, Dulbecco's modified Eagle medium (DMEM) and all analytical grade chemicals were from Himedia (India). Human cervical cancer ME-180 and Human Embryonic Kidney (HEK-293) cell lines obtained from National Centre for Cell Science (NCCS), Pune, India were used in this study.

\section{Nanoparticles synthesis}

Chitosan-TPP nanoparticles were prepared according to earlier reported method with modifications (Woranuch and Yoksan 2013). Briefly, chitosan solution $(1 \mathrm{mg} / \mathrm{ml})$ was prepared by agitating chitosan flakes with aqueous acetic acid $(1 \% \mathrm{v} / \mathrm{v})$ solution at ambient temperature overnight. Different concentrations (conc.) of TPP $(0.125,0.20,0.25$, $0.5,1 \mathrm{mg} / \mathrm{ml}$ ) were added slowly to the chitosan solution with constant stirring to obtain CS-TPP nanoparticles with optimum size properties. Nanoparticles were formed as a result of interaction between the negative groups of TPP and the positively charged amino groups of CS. For the preparation of FA-loaded CS-TPP nanoparticles, FA was added to the chitosan solution before TPP and effect of varying FA conc. $(5,10,20,40$ and $80 \mu \mathrm{M})$ was assessed on loaded nanoparticles' morphology.

\section{Evaluation of ferulic acid encapsulation and solubility}

FA/CS-TPP NPs were centrifuged at $14,000 \mathrm{rpm}$ for $30 \mathrm{~min}$ and the pellet was freeze-dried (Merlina et al. 2012). Supernatant was collected and the amount of nonencapsulated FA was measured spectrometrically (Lasany double beam LI-2800) at $319 \mathrm{~nm}$. The percentage encapsulation efficiency (\%EE) and loading capacity (\%LC) of FA were calculated using equations given below:

$$
\begin{aligned}
& \% \mathrm{EE}=(\text { Weight of initial FA loaded } \\
& - \text { weight of free FA/Weight of initial FA loaded }) \times 100 \\
& \% \text { LC }=(\text { Weight of initial FA loaded } \\
& \text { - weight of free FA/Total weight of nanoparticles }) \times 100
\end{aligned}
$$

Solubility of FA/CS-TPP NPs was tested by dissolving $1 \mathrm{mg}$ of dried pellet in $1 \mathrm{ml}$ acetic acid $(1 \% \mathrm{v} / \mathrm{v})$ and water 
followed by vortexing it for $5 \mathrm{~min}$. The mixtures were then kept in a magnetic stirrer for $12 \mathrm{~h}$ and the percent enhancement in solubility was recorded using native FA as control.

\section{Physicochemical characterization of nanoparticles}

\section{Morphology and size measurement}

Field-emission scanning electron microscopy (FESEM; Quanta 200F Model, FEI, Netherland) was used to examine the morphology of nanoparticles by fixing them onto glass slides and sputter coated (sputter coater: Biotech SC005, Switzerland) with gold for $1 \mathrm{~min}$. CSTPP as well as FA-loaded CS-TPP nanoparticles' diameters were measured over 50-100 different points of FESEM images using image $\mathrm{J}$ analyzer software. Zeta potential values of the synthesized nanoparticles were recorded using Zetasizer (Malvern Nano ZS; Malvern Instruments Ltd, UK).

\section{Fourier transformation infrared spectroscopy}

The Fourier transform infrared (FTIR) spectra of the nanoparticles were analyzed by an IR spectrometer (FTIR; Thermo Nicolet Nexus 6700, US). Average values of 32 scans were recorded with $4000-400 \mathrm{~cm}^{-1}$ wavelength scanning range and resolution of $4 \mathrm{~cm}^{-1}$ for each sample.

\section{Thermogravimetric and derivative thermogravimetric analyses}

Thermogravimetric and derivative thermogravimetric analyses of native FA and nanoparticles preparations (CSTPP, FA/CS-TPP NPs) were performed in a simultaneous DTA-TG Apparatus (EXSTAR, TG/DTA 6300). Samples (2-8 mg) were heated from 20 to $500{ }^{\circ} \mathrm{C}$ at a scanning rate of $10^{\circ} \mathrm{C} / \mathrm{min}$. Nitrogen was used as the purge gas at a flow rate of $20 \mathrm{ml} / \mathrm{min}$.

\section{Analysis of anticancer potential}

ME-180 cells were maintained in McCoy's modified medium supplemented with $10 \%$ FBS, $1 \%$ glutamine, $100 \mathrm{U} / \mathrm{ml}$ penicillin and $100 \mu \mathrm{g} / \mathrm{ml}$ streptomycin at $37{ }^{\circ} \mathrm{C}$ in $5 \% \mathrm{CO}_{2}$ atmosphere. Cells $\left(3 \times 10^{4} /\right.$ well $)$ were seeded in 96-well plates and treated with $10 \mu \mathrm{L}$ of each formulations; native FA $(5,10,20,40$ and $80 \mu \mathrm{M})$, CS-TPP and FA/CS-TPP NPs followed by MTT assay at $540 \mathrm{~nm}$ (Kyoungho et al. 2001). Untreated cells growing in culture media only were taken as control. The cell viability was calculated as:
Cell viability $(\%)=\mathrm{OD}_{540 \mathrm{~nm}}($ test samples $) / \mathrm{OD}_{540 \mathrm{~nm}}($ control $)$ $\times 100$

\section{Visualization of apoptotic morphological changes}

The control, native FA and FA/CS-TPP NPs treated cells were seeded in 6 -well plate $\left(3 \times 10^{4} /\right.$ well $)$ and incubated in $\mathrm{CO}_{2}$ incubator for $24 \mathrm{~h}$. The cells were immediately washed in PBS and observed using inverted phase contrast microscope (CarlZeiss, Axiovert 25, Germany). For FESEM analysis, cancer cells were fixed onto glass slide by $2.5 \%$ glutaraldehyde PBS solution for $4 \mathrm{~h}$ and visualized. For further validation of apoptotic morphological changes, the cells were dual stained with acridine orange (AO) and ethidiumbromide (EtBr) in 1:1 ratio and visualized using fluorescent microscope (CarlZeiss, Axiovert 25, Germany).

\section{Cytocompatibility evaluation}

Each test formulation ( $40 \mu \mathrm{M}$ native FA, unloaded CS-TPP NPs and $40 \mu \mathrm{M}$ FA/CS-TPP NPs) was tested for its cytocompatibilty by means of HEK-293 cells using a previously described protocol (Vashisth et al. 2015). Untreated cells were used as control; MTT assay and FESEM analysis (as described for ME-180 cells) were carried out following $24 \mathrm{~h}$ of cell seeding.

\section{Statistical analysis}

All measurements were performed in three independent experiments; data were expressed as mean value \pm standard deviation. The significance of differences $(p<0.05)$ among the corresponding mean values was determined using one-way analysis of variance (ANOVA).

\section{Results and discussion}

\section{Nanoparticles synthesis, morphology and encapsulation of ferulic acid}

The ratio between CS and TPP is critical in controlling the size as well as size distribution of NPs thereby affecting their biological performance (Pan et al. 2002). Different concentrations of TPP were used to find the optimum CSTPP ratio so as to obtain nanoparticles with narrow size distribution, before the FA encapsulation. CS-TPP weight ratio of 5/1 produced most compact nanoparticles with zeta potential value $44.2 \mathrm{meV}$ and average particle diameter of $57.5 \pm 27.6 \mathrm{~nm}$. FESEM images of CS-TPP NPs at $5 / 1$ weight ratio and their corresponding size distribution histogram are presented in Fig. 1. This ratio of 5/1 was 

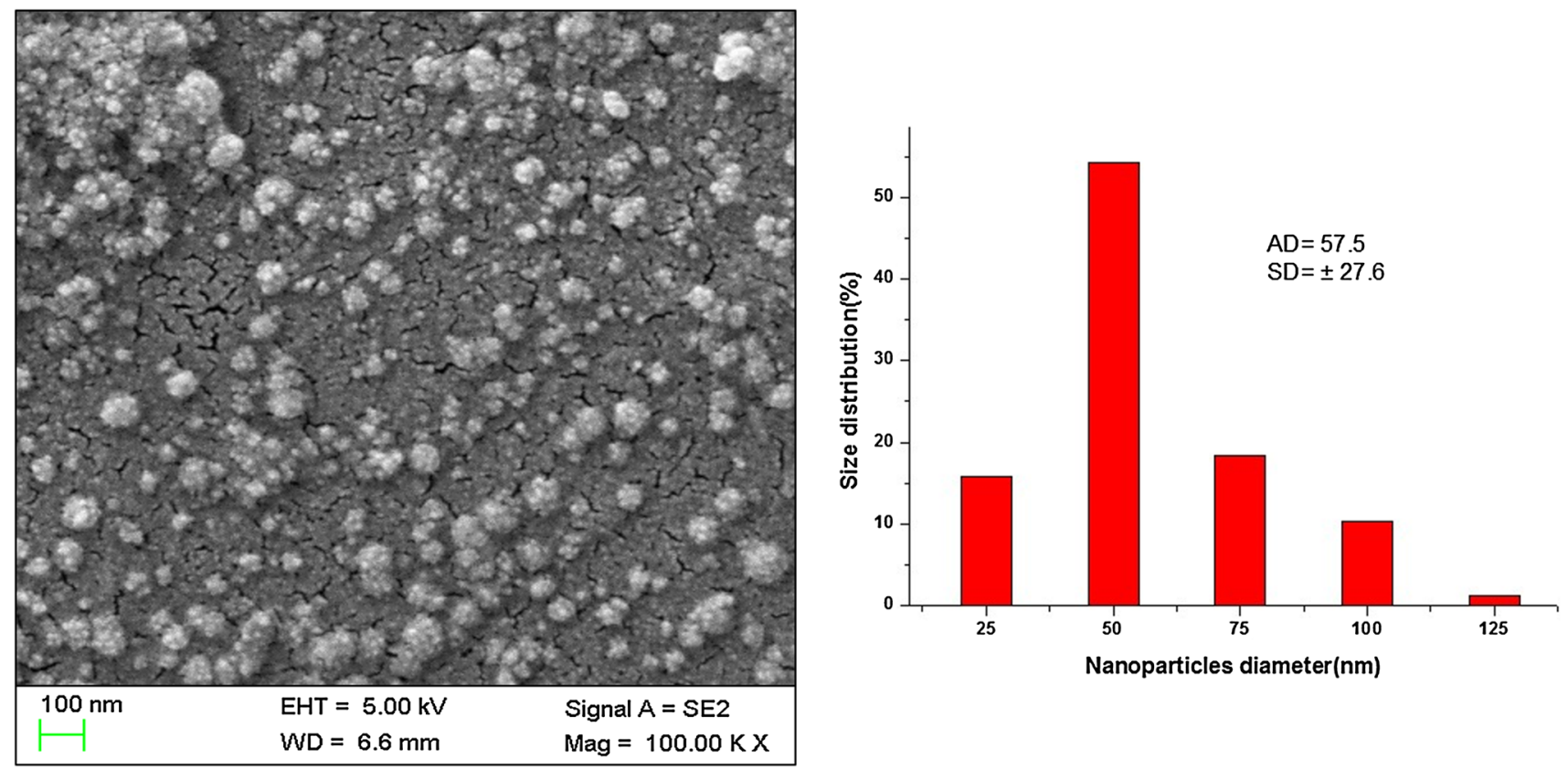

Fig. 1 FESEM micrographs of CS-TPP NPs (5/1 wt ratio) along with their diameter distribution histogram. Microscopic magnification $\times 100 \mathrm{~K}$, Scale bar $100 \mathrm{~nm}$. $S D$ standard deviation, $n=3 ; A D$ average diameter

Table 1 Average nanoparticles diameter, encapsulation efficiency and loading capacity of different test formulations

\begin{tabular}{llll}
\hline FA/CS-TPP $(\mu \mathrm{M}$ FA) & NPs Avg Dia $(\mathrm{nm})$ & EE of FA $(\%)$ & LC of FA $(\%)$ \\
\hline CS-TPP (5/1) & $57.5 \pm 27.6$ & - & - \\
FA/CS-TPP (5) & $48.3 \pm 13.8$ & - & - \\
FA/CS-TPP (10) & $67.4 \pm 23.4$ & $11.5 \pm 1.41$ & $4.4 \pm 1.3$ \\
FA/CS-TPP (20) & $89.3 \pm 14.6$ & $29.0 \pm 3.11$ & $11.3 \pm 0.9$ \\
FA/CS-TPP (40) & $125 \pm 44.7$ & $63.0 \pm 2.20$ & $32.5 \pm 3.4$ \\
FA/CS-TPP (80) & $1000-2000$ & $34.2 \pm 4.11$ & $32.9 \pm 2.1$ \\
\hline
\end{tabular}

$F A$ ferulic acid, NPs nanoparticles, $E E$ encapsulation efficiency, $L C$ loading capacity, $F A / C S-T P P$ ferulic acid-loaded chitosan-tripolyphosphate pentasodium, Avg average, Dia diameter selected for the preparation of FA-loaded nanoparticles. The average particle diameter of FA/CS-TPP NPs at varying FA concentrations along with their $\mathrm{EE}$ and $\mathrm{LC}$ is summarized in Table 1. Data showed that with increase in initial FA concentration EE also increased and reached to a maximum of $63.0 \pm 2.20 \%$ with $40 \mu \mathrm{M}$ FA. Similarly, LC also tended to increase as a function of initial FA content ranging from $4.4 \pm 1.3$ to $32.9 \pm 2.1 \%$. At $80 \mu \mathrm{M}$, a reduction in EE was observed while LC at this conc. remained almost equivalent to $40 \mu \mathrm{M}$ which could have occurred due to encapsulation limitation of chitosan at higher FA conc. The results obtained were in agreement with previous literature that reported encapsulation and loading of phenolic compound carvacrol into chitosan nanoparticles (Keawchaoon and Yoksan 2001). At $40 \mu \mathrm{M}$ FA conc. smooth and spherical FA-loaded nanoparticles with positive zeta potential value $\pm 22.5 \mathrm{meV}$ and average particle diameters of $125 \mathrm{~nm}$ (polydispersity index value
$=0.41$ ) were obtained (Fig. 2). Since no intercalated deposition of FA crystals was observed, it could be inferred that there was a uniform distribution of FA inside the CS-TPP NPs. The solubility of FA/CS-TPP NPs at $40 \mu \mathrm{M}$ was enhanced approximately by 28 and $25 \%$ in water and $1 \%$ acetic acid (v/v), respectively, at room temperature. The observed enhancement in solubility may be attributed the combined effect of interfacial adsorption and enhanced bioavailability of FA in the aqueous phase following encapsulation within CS. Interaction of phenolic phytochemicals with hydrophobic sites of nanoparticles through hydrogen bonds and hydrophobic forces is well established. Sufficient surface charges and suitable hydration property keep phenolic phytochemical encapsulated nanoparticles stable in aqueous system, which enhances the water solubility of phenolic phytochemicals ( $\mathrm{Li}$ et al. 2015). Significant decrease in zeta potential value of CSTPP NPs upon FA encapsulation from approximately +44 

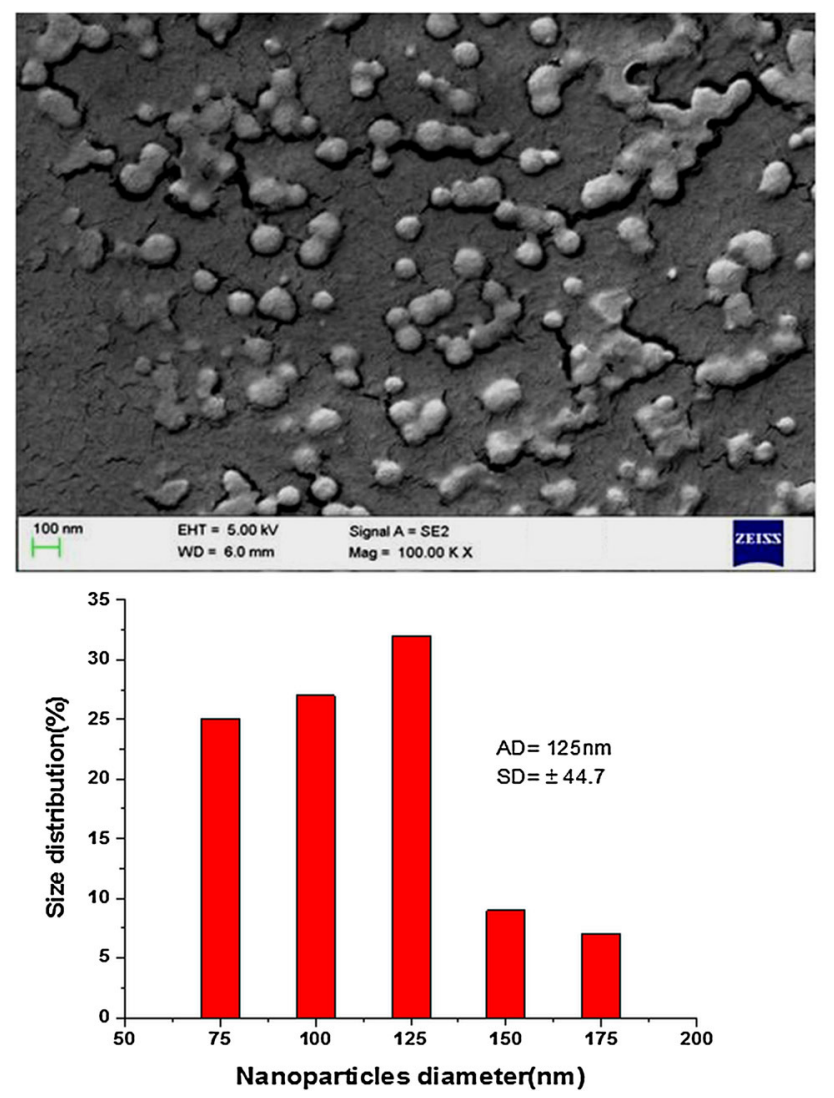

Fig. 2 FESEM micrographs of FA-loaded CS-TPP NPs (40 $\mu$ M FA) along with their diameter distribution histogram. Microscopic magnification $\times 100 \mathrm{~K}$, Scale bar $100 \mathrm{~nm}$. SD standard deviation, $n=3$; $A D$ average diameter

to $+22 \mathrm{meV}$ was observed. Zeta potential of a nanoparticle system expresses its stability in suspension through electrostatic repulsion between individual particles (Lee et al. 2010). So, the decrease in zeta potential of above two formulations could be in part attributed to the retardation of electrophoretic mobility of the nanoparticles as a result of their size enhancement upon FA encapsulation. Neutralization of some of the positive surface charges of CSTPP NPs might have also occurred due to adsorption of FA onto nanoparticles surface. Similar reduction in the zeta potential and subsequent size enhancement in L-ascorbic acid-loaded chitosan nanoparticles were reported earlier (Jang and Lee 2008). At $10 \mu \mathrm{M}$ FA concentration, smaller particles bound together to form random clusters were observed (Supplementary Fig. 1). However, at higher FA concentration $(80 \mu \mathrm{M})$, crystalline particles in the size range of $1-2 \mu \mathrm{m}$ were detected (Supplementary Fig. 2). Thus, from the data obtained it was concluded that $40 \mu \mathrm{M}$ conc. of FA was optimum to obtain fairly spherical and evenly distributed FA/CS-TPP NPs with a higher encapsulation efficiency for the present work.

\section{Fourier transformation infrared spectroscopy analysis}

To study various secondary interactions (hydrogen bonding, hydrophobic interactions and electrostatic forces) between the active groups of encapsulated FA and CS-TPP, FTIR spectra were analyzed. Characteristic peaks of native FA were observed at $3436 \mathrm{~cm}^{-1}$ (-OH stretching), $2249 \mathrm{~cm}^{-1}$ (C-H bond stretching), $1568,1411 \mathrm{~cm}^{-1}(\mathrm{C}=\mathrm{C}$ aromatic ring) as shown in Fig. 3a. The data were in accordance with the FTIR peaks reported earlier for FA (Ronald 2012). The FTIR spectra of cross-linked CS-TPP and FA/CS-TPP NPs are shown in Fig. 3b. For CS-TPP nanoparticles, a characteristic band at $3394 \mathrm{~cm}^{-1}$ is attributed to $-\mathrm{NH}_{2}$ and $-\mathrm{OH}$ groups stretching vibration, 1636 (amide II), $1384 \mathrm{~cm}^{-1}$ $\left(\mathrm{C}_{3}-\mathrm{O}\right)$ and $1073 \mathrm{~cm}^{-1}\left(\mathrm{C}_{6}-\mathrm{O}\right)$ as reported earlier (Vimal et al. 2013). For FA/CS-TPP NPs, increased peak intensity at each bond stretching resulted in shifting of peak values. A new peak was observed at $2437 \mathrm{~cm}^{-1}$, while the peaks at 3394 and $549 \mathrm{~cm}^{-1}$ were shifted to 3225 and $651 \mathrm{~cm}^{-1}$, respectively, due to hydrogen bonding between $-\mathrm{OH}$ and $\mathrm{NH}_{3}{ }^{+}$group of $\mathrm{CS}$ and $-\mathrm{OH}$ and $-\mathrm{C}=\mathrm{O}$ active groups of $\mathrm{FA}$ (Sofia et al. 2008; Liu et al. 2013). The presence of common peak at $1411 \mathrm{~cm}^{-1}$ corresponding to $\mathrm{C}=\mathrm{C}$ aromatic ring in both native FA and FA/CS-TPP indicates their interaction. Also, the band at $3436 \mathrm{~cm}^{-1}$ in case of native FA has been broadened and shifted to $3225 \mathrm{~cm}^{-1}$ signifying a possible $\mathrm{O}-\mathrm{H}-\mathrm{O}$ bonding occurring between native FA and FA/CSTPP. The FTIR spectra analyses thus indicate the formation of a copolymer, suggesting the successful loading of FA into the CS-TPP nanoparticles (Fig. 3c).

\section{Thermogravimetric and derivative thermogravimetric analyses}

Thermal properties of samples were determined by thermogravimetric analysis (TGA) to investigate the mass loss of a sample upon heating at a given heating rate. The TGA curves of FA, CS-TPP and FA/CS-TPP NPs are shown in Fig. 4a. FA exhibited a single stage weight loss, with decomposition starting at $173{ }^{\circ} \mathrm{C}$, while CS-TPP NPs exhibited decomposition in two stages. An initial weight loss of CS-TPP NPs between 150 and $250{ }^{\circ} \mathrm{C}$ may be attributed to dehydration of the saccharide rings, while the second stage decomposition ranging from 275 to $450{ }^{\circ} \mathrm{C}$ resulted from complex processes including depolymerization and decomposition of the acetylated and deacetylated units of polymer (Sindhu and Abraham 2008). Similarly for FA-loaded CS-TPP, the initial weight loss was observed at 80-100 ${ }^{\circ} \mathrm{C}$ due to evaporation of water. Major weight loss started at approximately $200{ }^{\circ} \mathrm{C}$ and continued linearly up to $500{ }^{\circ} \mathrm{C}$. The decrease in decomposition temperature of FA/CS-TPP NPs, over unloaded CS-TPP NPs might be due 

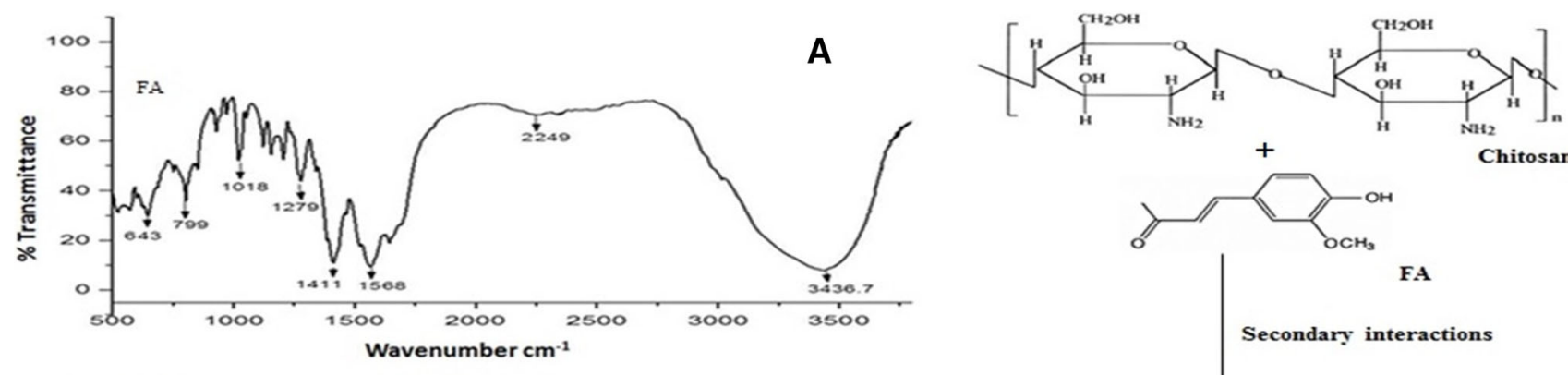

Chitosan
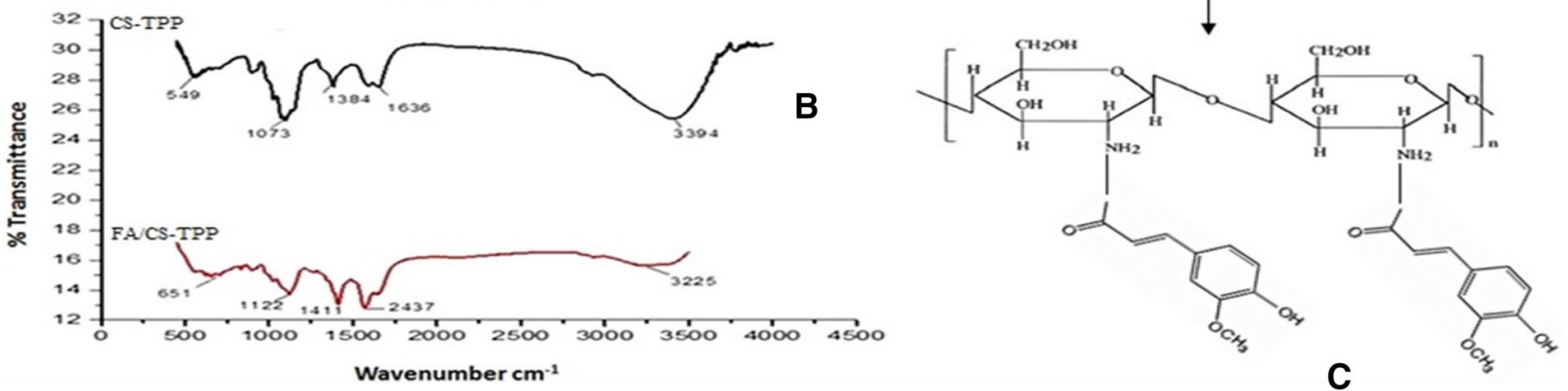

Fig. 3 FTIR spectra a FA, b CS-TPP and c FA-loaded chitosan-TPP nanoparticles
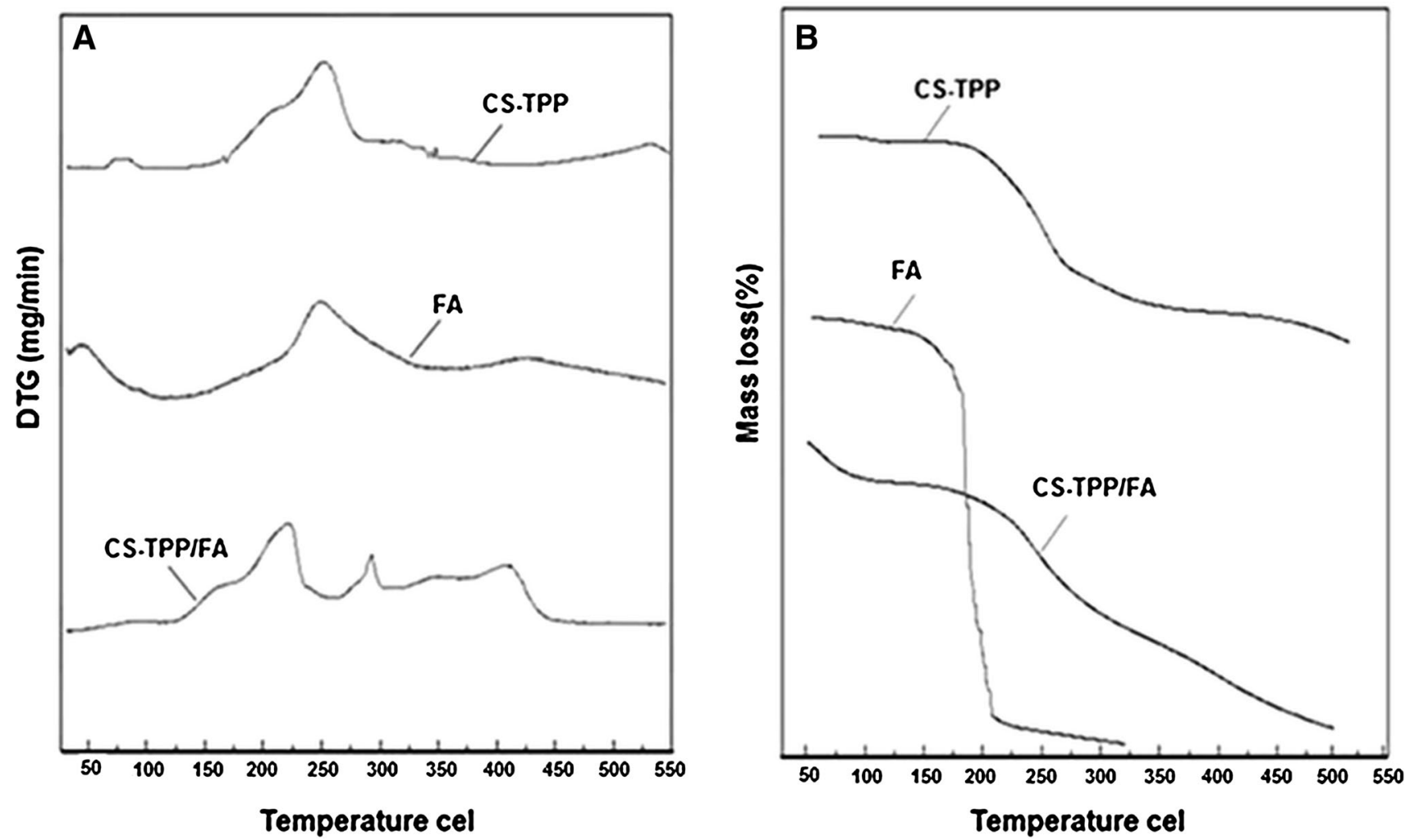

Fig. 4 Thermograms of native FA, CS-TPP NPs and FA-loaded CS-TPP NPs a TGA, b DTG

to the reduction in crystalline structure of CS following FA encapsulation. However, this reduction in decomposition temperature had negligible effect on stability and integrity of FA-loaded nanoparticles structure as indicated by derivative thermogravimetric analyses (Fig. 4b). The thermogravimetric analyses thus indicated that FA could be successfully loaded into CS-TPP nanoparticles, to increase its thermal resistance. 
The derivative thermogravimetric analyses of the FA and CS-TPP nanoparticles showed peak maximum decomposition at 247 and $272{ }^{\circ} \mathrm{C}$, respectively; while FAloaded CS-TPP nanoparticles exhibited decomposition temperatures approximately at 220, 290 and $430{ }^{\circ} \mathrm{C}$ (Fig. 4b). The distribution of peak maxima in encapsulated FA may be a result of its interaction with polymeric components of CS.

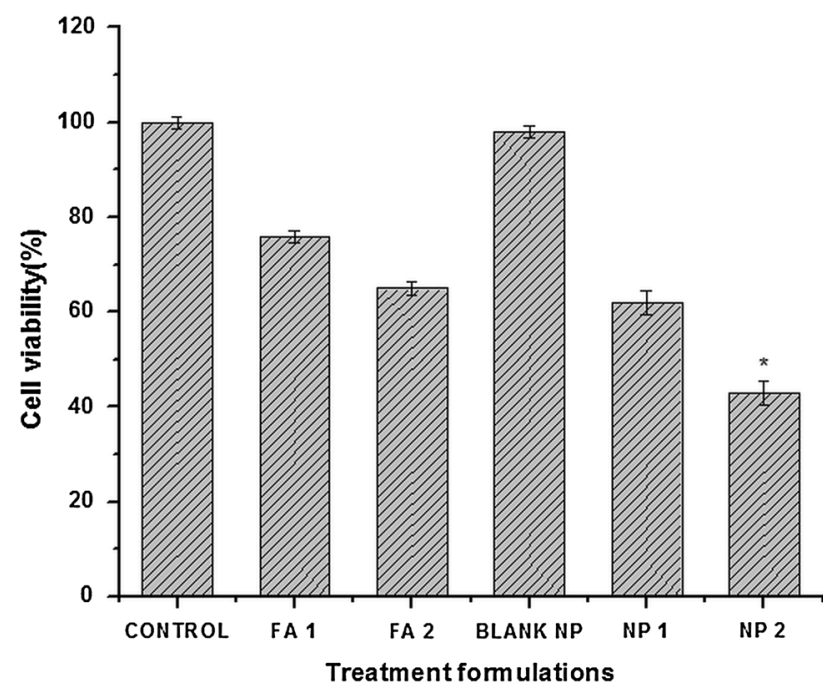

Fig. 5 MTT viability assay for ME-180 cells treated with different test formulations after $24 \mathrm{~h}$ at $540 \mathrm{~nm}$. Error bars represent mean \pm standard deviation for three independent experiments $(n=3), * p<0.05$ indicates statistical significant difference as compared to control
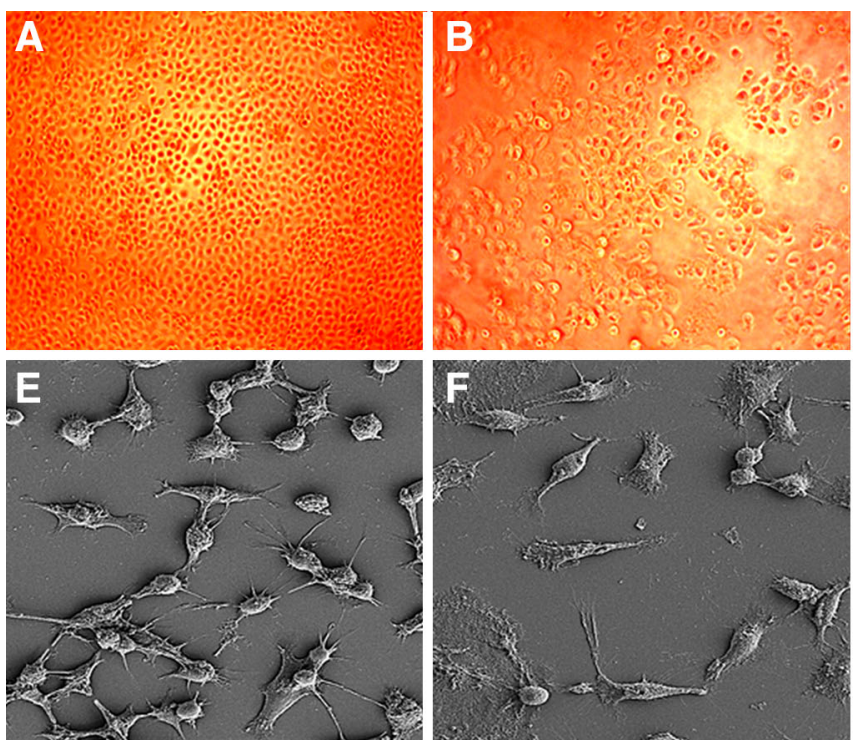

Fig. 6 ME-180 cell lines showing morphological effects through phase contrast micrographs and its corresponding scanning electron micrographs (a, e) control cells $(\mathbf{b}, \mathbf{f})$ cells treated with $40 \mu \mathrm{M}$ native

\section{In vitro cytotoxicity analysis}

ME-180 cervical cancer cell lines were tested for growth inhibition under different formulations, viz., $20 \mu \mathrm{M}$ native FA (FA1), $40 \mu \mathrm{M}$ native FA (FA2), unloaded CS-TPP NPs, $20 \mu \mathrm{M}$ (NP1) and $40 \mu \mathrm{M}$ FA/CS-TPP (NP2) keeping untreated cells as control (Fig. 5). As indicated, $40 \mu \mathrm{M}$ FA/CS-TPP NPs caused maximum inhibition $(57 \%)$ of cell proliferation as compared to other formulations, this concentration was also found to yield most stable formulation of encapsulated nanoparticles. Previous studies on FA-treated ME-180 cervical cancer cell lines have indicated increased intracellular ROS levels along with inhibition of cell growth, increased lipid peroxidation and profound induction of cell apoptosis, in combination with gamma radiations (Subburayan et al. 2011). Literature reports that chitosan nanoparticles owing to their positive surface charges adsorb with a high affinity onto the negatively charged tumor cell membrane (Qi et al. 2005). In the present investigation, FA/CS-TPP NPs would have bind to the tumor cell membrane via electron interactions, entered the cell through endocytosis and release the encapsulated FA into cytosol (endosomal burst). Due to increased bioavailability, FA exhibits its antitumor effect by influencing the mitochondrial activity and antioxidant status of the cell disrupting the cell organelle which would eventually lead to cell death. In our earlier reports, FA from Parthenium hysterophorus and FA encapsulated nanofibers were found to exhibit anticancer potential against different cell lines (Panwar et al. 2015, Vashisth et al. 2015). Zhou et al. (2007) reported the $27 \%$
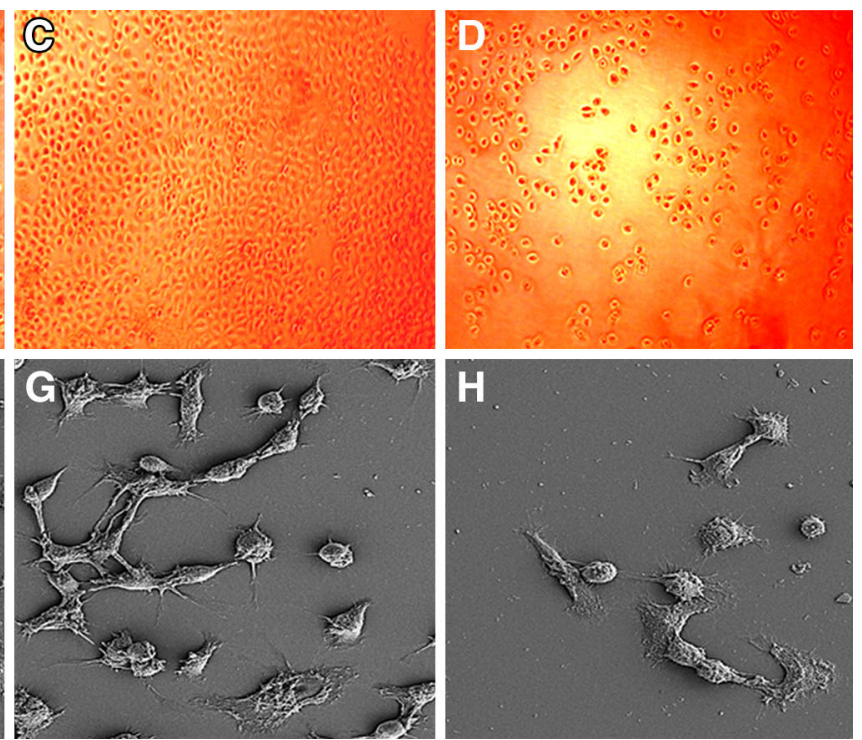

FA $(\mathbf{c}, \mathbf{g})$ cells treated with CS-TPP NPs $(\mathbf{d}, \mathbf{h})$ cells treated with $40 \mu \mathrm{M}$ FA-loaded CS-TPP NPs upon $24 \mathrm{~h}$ of incubation. Scale bar $10 \mu \mathrm{m}$, microscopic magnification $\times 10 \mathrm{~K}$ 
inhibition of HeLa cervical cancer cell lines when treated with $500 \mathrm{mg} / \mathrm{L}$ chitosan nanoparticles. Decrease in mitochondrial membrane proteins by chitosan nanoparticles has also been reported earlier (Qi et al. 2005). From our data it could be inferred that $40 \mu \mathrm{M}$ FA/CS-TPP NPs displayed an enhanced cytotoxicity against ME-180 cervical cancer cell lines which could be attributed to the synergistic effect of both FA and CS-TPP NPs causing the disruption of cancer cell membrane integrity and mitochondrial damage.

\section{Apoptotic morphological changes}

To observe the cytotoxic effect of different formulations on cell morphology, the cells were examined by phase contrast and scanning electron microscopy. Data showed that the
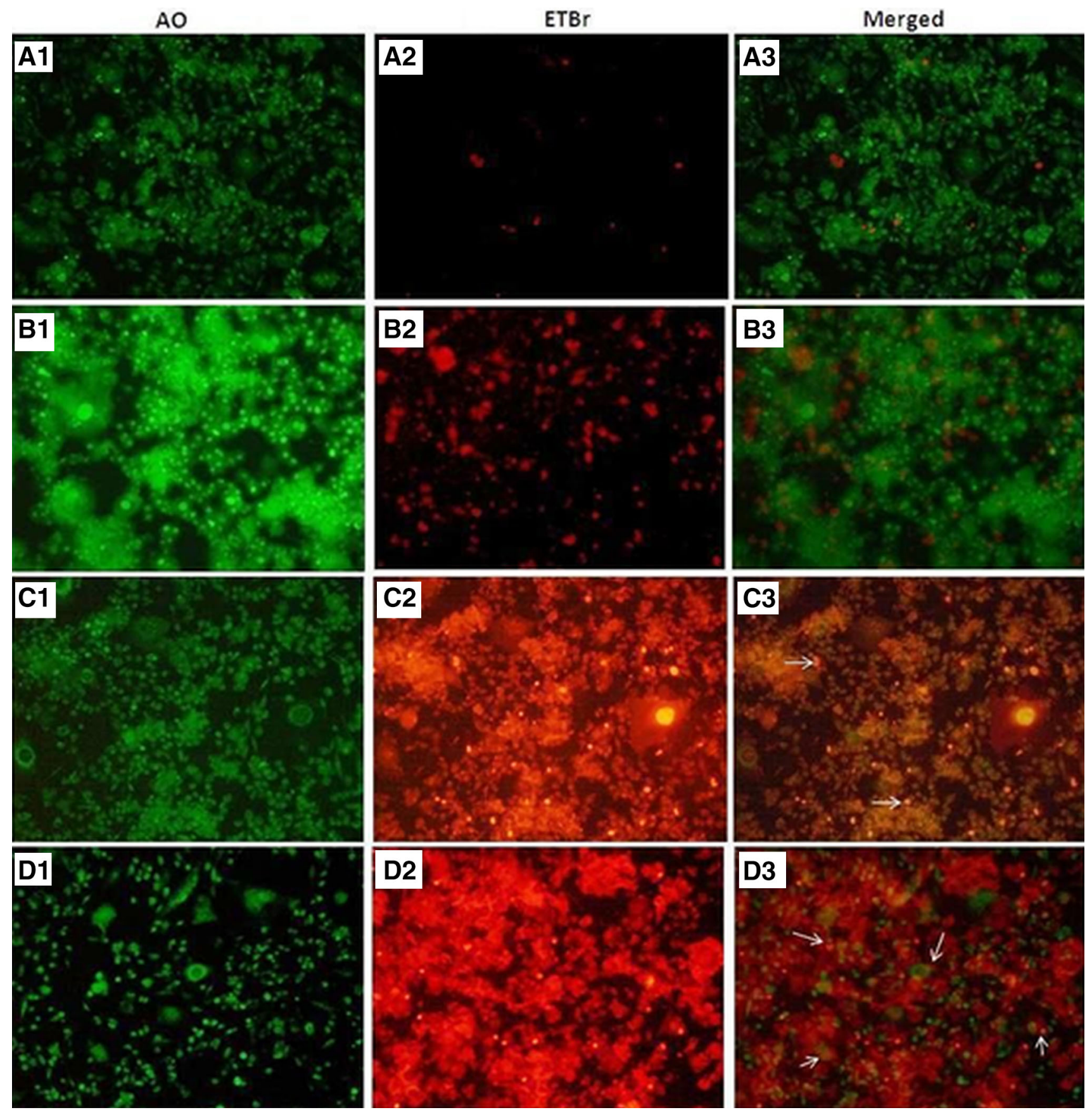

Fig. 7 Fluorescent micrographs of AO:EtBr stained ME-180 cells $\left(\mathbf{A}^{\mathbf{1}}-\mathbf{A}^{\mathbf{3}}\right)$ control cells $\left(\mathbf{B}^{\mathbf{1}}-\mathbf{B}^{\mathbf{3}}\right)$ CS-TPP NPs treated $\left(\mathbf{C}^{\mathbf{1}}-\mathbf{C}^{\mathbf{3}}\right) 40 \mu \mathrm{M}$ native FA treated $\left(\mathbf{D}^{\mathbf{1}}-\mathbf{D}^{\mathbf{3}}\right)$ FA/CS-TPP NPs treated after $24 \mathrm{~h}$ incubation. Morphological signs such as chromatin condensation and damaged wrinkled cells are marked with white arrows. Microscopic magnification $\times 100 \mathrm{~K}$ 
cells treated with $40 \mu \mathrm{M}$ FA/CS-TPP NPs for $24 \mathrm{~h}$ displayed morphological characteristics such as higher cell shrinkage, cytoplasmic condensation, and irregularity in shape, suggesting that FA-loaded chitosan nanoparticles induce apoptotic cell death in the ME-180 cells (Fig. 6d, h). The morphology and growth of cancer cells cultured on unloaded CS-TPP NPs were only negligibly affected as compared to control (Fig. 6c, g). Significant reduction in cancer cell number/growth was observed in the FESEM micrographs in the cells treated with FA/CS-TPP NPs (Fig. 6h). To further confirm the induction of apoptosis, treated cells were visualized by fluorescence microscopy following treatment with 1:1 ratio of $\mathrm{AO} / \mathrm{EtBr}$, which allow differentiation of dead and viable cells by staining DNA. Cells with intact membranes fluoresce green due to $\mathrm{AO}$ staining while $\mathrm{EtBr}$ stains cells with damaged membranes which exhibit orange fluorescence due to DNA intercalation of both stains. As depicted in Fig. 7, ME-180 cells treated with unloaded CS-TPP NPs did not show any retardation of cell proliferation, similar to control cells. On the other hand, cells treated with native FA and FA/CSTPP NPs have undergone considerable damage. Morphological signs such as chromatin condensation and damaged wrinkled cells indicated encapsulated FA-induced apoptosis to destroy cancer cells (Fig. $7 \mathrm{D}^{3}$ ). Results obtained suggested the role of CS-TPP NPs in guiding the encapsulated FA more precisely towards a physiological target hence complementing to generate stronger and more efficient antiproliferative action of FA/CS-TPP NPs as compared to native FA. Earlier literature reported the antitumor and antiproliferative nature of encapsulated FA in bio- compatible polymers against different cell lines and dosedependent apoptosis of cancer cells treated with chitosan nanoparticles leading to cell death induction (Sofia et al. 2008; Woranuch and Yoksan 2013; Qi et al. 2005).

\section{Cytocompatibility evaluation}

To verify the potential applications of FA-loaded nanoparticles as a clinical therapeutic against cancer cells, its cytocompatibility was tested on HEK-293 cells. Surface morphology of the cells seeded on native FA and FA/ CS-TPP NPs are presented in Fig. 8. Negligible changes in cell adherence and morphology could be visible in the cells treated with FA/CS-TPP NPs (Fig. 8c) as compared to control cell's matrix (Fig. 8a). In contrast, complete cell disruption and significant change in surface morphology were observed (Fig. 8b) for cells treated with $40 \mu \mathrm{M}$ native FA which may be attributed to its free radical scavenging ability (Ou and Kwok 2004). Studies have revealed that phenolic compounds such as FA at higher concentrations may act as pro-oxidant interacting with transition metal ions found in biological systems which lead to oxidative damage of normal cellular components (Maurya and Devasagayam 2010; Galati and O'Brien 2004). Our research group too has reported that the viability of non-cancerous HEK-293 cell lines was lower in presence of native FA in comparison with the nanofiber encapsulated FA (Vashisth et al. 2015). Data obtained in our investigation imply superior cytocompatibilty of FA/ CS-TPP NPs as compared to native FA at the same concentration $(40 \mu \mathrm{M})$.
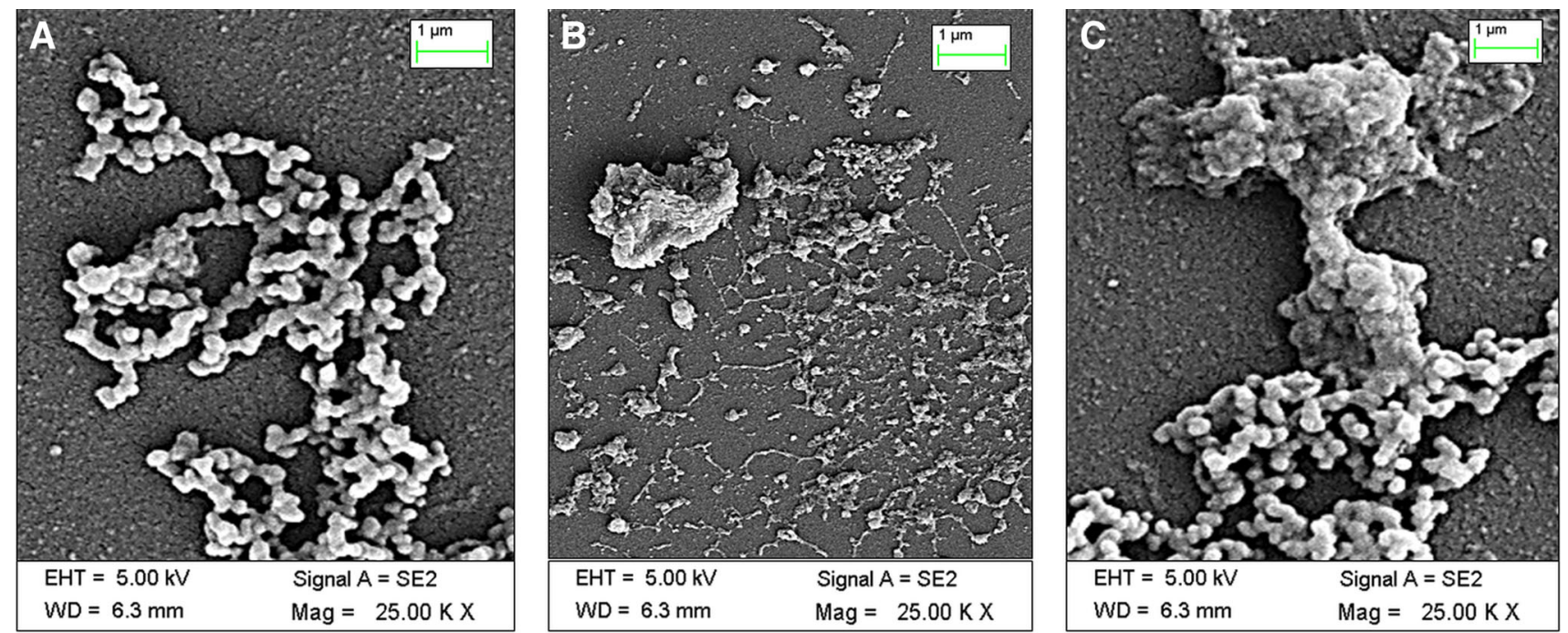

Fig. 8 SEM micrographs of HEK-293 cells (a) control (b) cell treated with $40 \mu \mathrm{M}$ native FA (c) cells treated with $40 \mu \mathrm{M}$ FA-loaded CS-TPP NPs incubated for $24 \mathrm{~h}$. Scale bar $1 \mu \mathrm{m}$, microscopic magnification $\times 25 \mathrm{~K}$ 


\section{Conclusions}

In this study, we encapsulated FA into CS-TPP nanoparticles to develop a polymeric nanocarrier with enhanced thermal degradation range. Secondary interactions of FA with CS-TPP nanoparticles were studied using FTIR, suggesting its successful encapsulation. Analyses of nanoparticles morphology indicated the formation of smooth and fairly spherical FA/CS-TPP NPs, with high encapsulation efficiency. MTT assay revealed strong antiproliferative activity of FA-loaded chitosan-TPP nanoparticles due to apoptotic induction as shown by morphological analyses. The results so obtained suggest that the encapsulation of FA into CS-TPP nanoparticles may enhance its cytocompatibilty, solubility and anticancer potential against ME-180 cell lines, thereby presenting it as a potent therapeutic agent for medicine and clinical usage.

Acknowledgments Authors are thankful to Indian Institute of Technology Roorkee, UK, India, for providing the infrastructural facilities to carry out the research work.

\section{Compliance with ethical standards}

Conflict of interest The authors declare no conflicts of interest.

Open Access This article is distributed under the terms of the Creative Commons Attribution 4.0 International License (http:// creativecommons.org/licenses/by/4.0/), which permits unrestricted use, distribution, and reproduction in any medium, provided you give appropriate credit to the original author(s) and the source, provide a link to the Creative Commons license, and indicate if changes were made.

\section{References}

Akihisa T, Yasukawa K, Yamaura M, Ukiya M, Kimura Y, Shimizu N, Arai K (2000) Triterpene alcohol and sterol ferulates from rice bran and their anti-inflammatory effects. J Agric Food Chem 48:2313-2319

Ellenson LH, Wu TC (2004) Focus on endometrial and cervical cancer. Cancer Cell 5:533-538

Galati G, O'Brien PJ (2004) Potential toxicity of flavonoids and other dietary phenolics: significance for their chemopreventive and anticancer properties. Free Radic Biol Med 37(3):287-303

Garg AK, Buchholz TA, Aggarwal BB (2005) Chemosensitization and radiosensitization of tumors by plant polyphenols. Antioxid Redox Signal 7:1630-1647

Hu B, Pan C, Sun Y, Hou Z, Ye H, Zeng X (2008) Optimization of fabrication parameters to produce chitosan-tripolyphosphate nanoparticles for delivery of tea catechins. J Agric Food Chem 56:7451-7458

Jang KI, Lee HG (2008) Stability of chitosan nanoparticles for 1 -ascorbic acid during heat treatment in aqueous solution. J Agric Food Chem 56:1936-1941

Janicke B, Hegardt C, Krogh M, Onning G, Akesson B, Cirenajwis HM, Oredsson SM (2011) The antiproliferative effect of dietary fiber phenolic compounds ferulic acid and p-coumaric acid on the cell cycle of Caco-2 cells. Nutr Cancer 63(4):611-622
Kampa M, Alexaki VI, Notas G, Nifli AP, Nistikaki A, Hatzoglou A, Bakogeorgou E, Kouimtzoglou E, Blekas G, Boskou D, Gravanis A, Castanas E (2004) Antiproliferative and apoptotic effects of selective phenolic acids on T47D human breast cancer cells: potential mechanisms of action. Breast Cancer Res 6:63-74

Kayahara H, Miao Z, Fujiwara G (1999) Synthesis and biological activities of ferulic acid derivatives. Anticancer Res 19:3763-3768

Keawchaoon L, Yoksan R (2001) Preparation, characterization and in vitro release study of carvacrol-loaded chitosan nanoparticles. Colloids Surf B Biointerfaces 84:163-171

Kyoungho S, Yun-Hee K, Inik C (2001) IFNK sensitizes ME-180 human cervical cancer cells to TNFK-induced apoptosis by inhibiting cytoprotective NF-UB activation. FEBS Lett 495:66-70

Lee JS, Kim GH, Lee HG (2010) Characteristics and antioxidant activity of Elsholtzia splendens extract-loaded nanoparticles. J Agric Food Chem 58:3316-3321

Li Z, Jiang H, Xu C, Gu L (2015) A review: using nanoparticles to enhance absorption and bioavailability of phenolic phytochemicals. Food Hydrocoll 43:153-164

Liu N, Chen XG, Park HJ, Liu CG, Liu CS, Meng XH, Yu LJ (2006) Effect of MW and concentration of chitosan on antibacterial activity of Escherichia coli. Carbohydr Polym 64:60-65

Liu J, Lu JF, Kan J, Tang Y, Jin C (2013) Preparation, characterization and antioxidant activity of phenolic acids grafted carboxymethyl chitosan. Int J Biol Macromol 62:85-93

Maurya DK, Devasagayam TPA (2010) Antioxidant and prooxidant nature of hydroxycinnamic acid derivatives ferulic and caffeic acids. Food Chem Toxicol 48:3369-3373

Merlina JP, Prasad NR, Shibli SMA, Mol S (2012) Ferulic acid loaded poly-d, 1-lactide-co-glycolide nanoparticles: systematic study of particle size, drug encapsulation efficiency and anticancer effect in non-small cell lung carcinoma cell line in vitro. Biomed Prev Nutr 2:69-76

Mori H, Kawabata K, Yoshimi N, Tanaka T, Murakami T, Okada T, Murai H (1999) Chemopreventive effects of ferulic acid on oral and rice germ on large bowel carcinogenesis. Anticancer Res 19:3775-3778

Munin A, Lévy FE (2011) Encapsulation of natural polyphenolic compounds; a review. Pharmaceutics 3:793-829

Ou S, Kwok KC (2004) Ferulic acid: pharmaceutical functions, preparation and applications in foods. J Sci Food Agric 84:1261-1269

Ou S, Li Y, Gao K (1999) A study on scavenging activity of wheat bran dietary fiber for free radical. Acta Nutr Sin 21:191-194

Pan Y, Li YJ, Zhao HY (2002) Bioadhesive polysaccharide in protein delivery system: chitosan nanoparticles improve the intestinal absorption of insulin in vivo. Int J Pharm 249(1-2):139-147

Panwar R, Sharma AK, Dutt D, Pruthi V (2015) Phenolic acids from Parthenium hysterophorus: evaluation of bioconversion potential as free radical scavengers and anticancer agents. Adv Biosci Biotechnol 6:11-17

Qi LF, Xu ZR, Li Y, Jiang X, Han XY (2005) In vitro effects of chitosan nanoparticles on proliferation of human gastric carcinoma cell line MGC803 cells. World J Gastroenterol 11(33):5136-5141

Ronald AH (2012) Principal component analysis of phenolic acid spectra. ISRN Spectrosc. doi:10.5402/2012/493203

Saija A, Tomaino A, Trombetta D, De Pasquale A, Uccella N, Barbuzzi T, Paolino D, Bonina F (2000) In vitro and in vivo evaluation of caffeic and ferulic acids as topical photoprotective agents. Int J Pharm 199:39-47

Salmaso S, Bersani S, Semenzato A, Caliceti P (2007) New cyclodextrin bioconjugates for active tumour targeting. J Drug Target 15:379-390 
Seiwert TY, Salama JK, Vokes EE (2007) The concurrent chemoradiation paradigm-general principles. Natl Clin Pract Oncol 4:86-100

Sindhu M, Abraham TE (2008) Characterisation of ferulic acid incorporated starch-chitosan blend films. Food Hydrocoll 22:826-835

Sofia P, Dimitrios B, Konstantinos A, Evangelos K, Manolis G (2008) Chitosan nanoparticles loaded with dorzolamide and pramipexole. Carbohydr Polym 73:44-54

Soobrattee MA, Neergheen VS, Luximon-Ramma A, Aruomab OI, Bahorun T (2005) Phenolics as potential antioxidant therapeutic agents. Mechanism and actions. Mutat Res 579:200-213

Subburayan K, Govindhasamy K, Nagarajan RP, Rajendran M (2011) Radiosensitizing effect of ferulic acid on human cervical carcinoma cells in vitro. Toxicol In Vitro 25:1366-1375

Vashisth P, Sharma M, Kumar N, Singh H, Panwar R, Pruthi PA, Pruthi V (2015) Antiproliferative activity of ferulic acidencapsulated electrospun PLGA/PEO nanofibers against MCF7 human breast carcinoma cells. 3 Biotech 5:303-315
Vemula PK, Li J, John G (2006) Enzyme catalysis: tool to make and break amygdalin hydrogelators from renewable resources: a delivery model for hydrophobic drugs. J Am Chem Soc 128:8932-8938

Vimal S, Majeed SA, Taju G (2013) Chitosan tripolyphosphate (CS/ TPP) nanoparticles: preparation, characterization and application for gene delivery in shrimp. Acta Trop 128:486-493

Wilson TA, Nicolosi RJ, Woolfrey B, Kritchevsky D (2007) Rice bran oil and oryzanol reduce plasma lipid and lipoprotein cholesterol concentrations and aortic cholesterol ester accumulation to a greater extent than ferulic acid in hypercholesterolemic hamsters. J Nutr Biochem 18:105-112

Woranuch S, Yoksan R (2013) Eugenol-loaded chitosan nanoparticles: I. Thermal stability improvement of eugenol through encapsulation. Carbohydr Polym 96:578-585

Zhou SH, Hong Y, Fang GJ (2007) Preparation, characterization and anticancer effect of chitosan nanoparticles. J Clin Rehab Tiss Engin Res 11(48):9688-9691 